\title{
Defective IgG2 response to Pneumovax in HIV seropositive patients
}

\author{
D J Unsworth, D Rowen, C Carne, C Sonnex, T Baglin, D L Brown
}

\begin{abstract}
Objective-To determine whether HIV (Human Immunodeficiency Virus) antibody positive adults are capable of mounting an effective immune response when immunized with polyvalent pneumococcal vaccine.

Design-28 patients (nine homosexual men, one bisexual man, three heterosexual females, 10 injecting drug abusers, five haemophiliacs) and 11 healthy volunteers, were immunised with Pneumovax II and titres of IgG1 and IgG2 specific antibody measured before and 1 month after immunisation. Magnitude of immune response was related to CD4 T-lymphocyte count at time of immunisation to establish whether responses are better in early disease.

Main outcome measures-Based on our data in healthy volunteers we defined an adequate response to Pneumovax II as a post immunisation IgG2 antibody level at least $50 \%$ greater than the pre immunisation level.

Results-The magnitude of the response was significantly higher in the normal volunteers $\quad(U=95 ; \quad p=0.0328)$. Adequate IgG2 responses were seen in $11 / 11$ normals but in only $14 / 28 \mathrm{HIV}$ seropositives $\left(\chi^{2}=8.58 ; \mathrm{p}<0.01\right)$. Poor responses were unrelated to the CD4 Tlymphocyte count at immunisation. Absolute IgG2 deficiency accounted for the poor response in only 1 HIV patient. Conclusion-50\% of HIV antibody seropositive individuals fail to mount adequate immune responses to polyvalent pneumococcal vaccine. Non responders are unlikely to be protected.
\end{abstract}

(Genitourin Med 1993;69:373-376)

Departments of

Clinical Immunology, Genitourinary Medicine, and Haematology Addenbrooke's Hospital D J Unsworth D Rowen C Carne C Sonnex

C Sonne

$T$ Baglin

D L Brown

Correspondence to: Dr D J Unsworth, Level 4E, Addenbrooke's Hospital, Hills Road, Cambridge CB2 2QQ UK.

Accepted for publication 17 June 1993 trials in this immunocompromised group. Indeed, vaccine failure has been reported in AIDS. ${ }^{1}$

Several lines of evidence indicate that protection against Streptococcus pneumoniae largely depends on the possession of specific IgG2 antibody. IgG2 is the major isotype produced in response to polysaccharide antigens in adults (before 10 years of age IgG2 responses are immature, and compensatory IgG1 responses are mounted). ${ }^{6}$ Absolute $^{7}$ or functional ${ }^{8}$ deficiency of IgG2 at any age is associated with susceptibility to infection with capsulated organisms, and the failure of patients with Hodgkin's disease to respond to PPPV was associated with low total levels of IgG2 pre immunisation. ${ }^{9}$ There have been many studies of the humoral response to PPPV in HIV infected persons, ${ }^{10}$ but we know of only one published study which measured the IgG2 subclass response in this context. ${ }^{11}$ Twenty five individuals with HIV related persistent generalised lymphadenopathy were immunised, and 16 of these failed to mount adequate specific IgG2 responses. These results beg the question whether IgG2 responses are impaired at all stages of infection. CD4 counts fall progressively with time in HIV infection and serve as a good index of disease progression. ${ }^{12} \mathrm{We}$ therefore related CD4 counts at the time of immunisation to the magnitude of the ensuing specific IgG2 response. Where poor IgG2 responses were found, we checked whether absolute IgG2 deficiency or a subclass switch to the more juvenile IgG1 response accounted for our results.

Patients and materials and methods Normal individuals are expected to have detectable levels of antibody even before immunisation as a consequence of subclinical exposure to Streptococcus pneumoniae. The normal pre immunisation specific IgG2 antiPneumovax range was established in $38 \mathrm{HIV}$ antibody negative blood donors. Response to intramuscular immunisation with $0.5 \mathrm{ml}$ Pneumovax II (Merck Sharp and Dohme Ltd, Hertfordshire) was assessed in 11 healthy members of hospital staff aged 24-51 years (four female). Responses were also assessed in $28 \mathrm{HIV}$ seropositive persons aged 21-48 years, including nine homosexual men, one bisexual man, three heterosexual females, 10 injecting drug abusers (five female), and five haemophiliacs. Pneumovax II was administered to the haemophiliacs by subcutaneous polyvalent pneumococcal polysaccharide vaccines (PPPV) is recommended by the Department Of Health (UK) ${ }^{4}$ and the Centre For Disease Control (Atlanta). ${ }^{5}$ However, efficacy has never been assessed by clinical 
IgG Specific Anti-Pneumovax

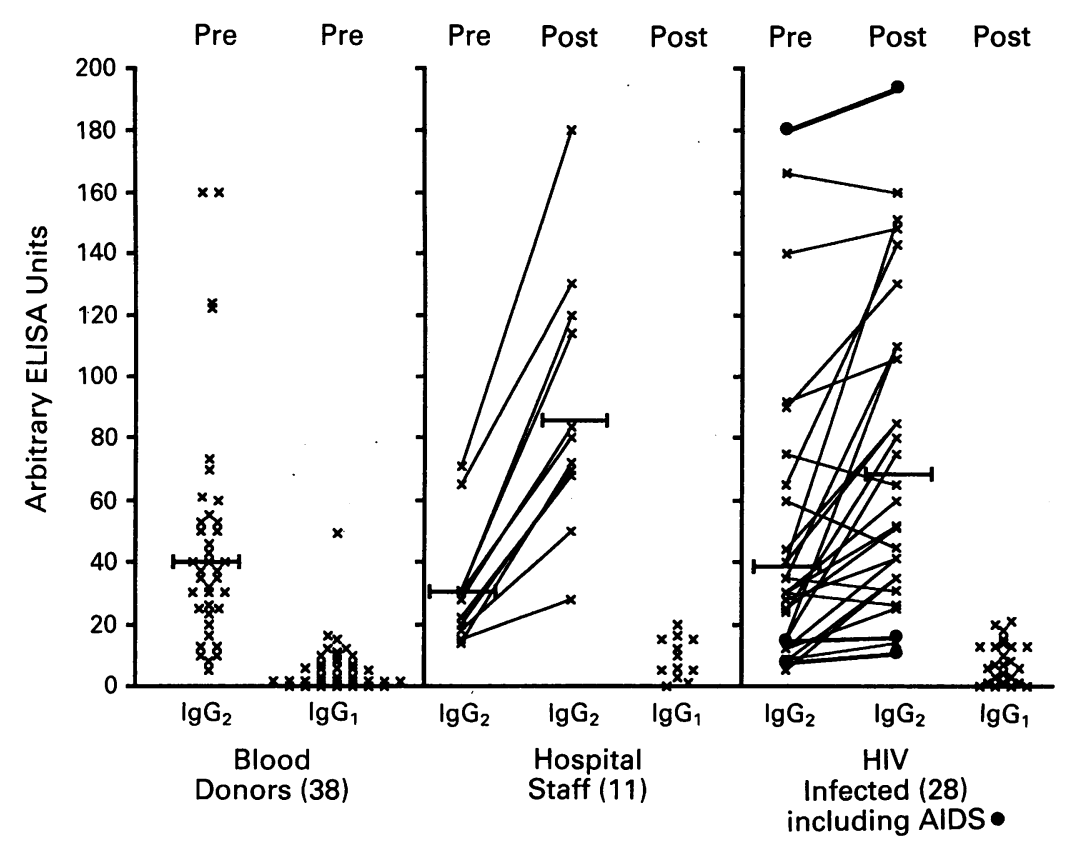

Figure 1 Titres of specific IgG1 and IgG2 antibody to Pneumovax II, pre and post immunisation. injection. One homosexual, one drug abuser, and the bisexual male, had AIDS that is CDC Stage IVc disease. All the other HIV seropositives were CDC Stages II or III. Studies in normal vaccinees showed that antibody responses measured at 2 and 4 weeks post vaccination were identical. Results at 4 weeks post vaccination are reported here.

We used Pneumovax II itself as substrate in our ELISA test for specific antibody. All incubation steps were at $25^{\circ} \mathrm{C}$. Nunc (Maxisorb) plastic plates were coated overnight with Pneumovax II (the undiluted vaccine contains $25 \mu \mathrm{g}$ of each of the 23 different pneumococcal polysaccharide types), diluted $1 / 100$ in $0 \cdot 1 \mathrm{M}$ bicarbonate/carbonate buffer $\mathrm{pH} 9 \cdot 6$. All subsequent incubations

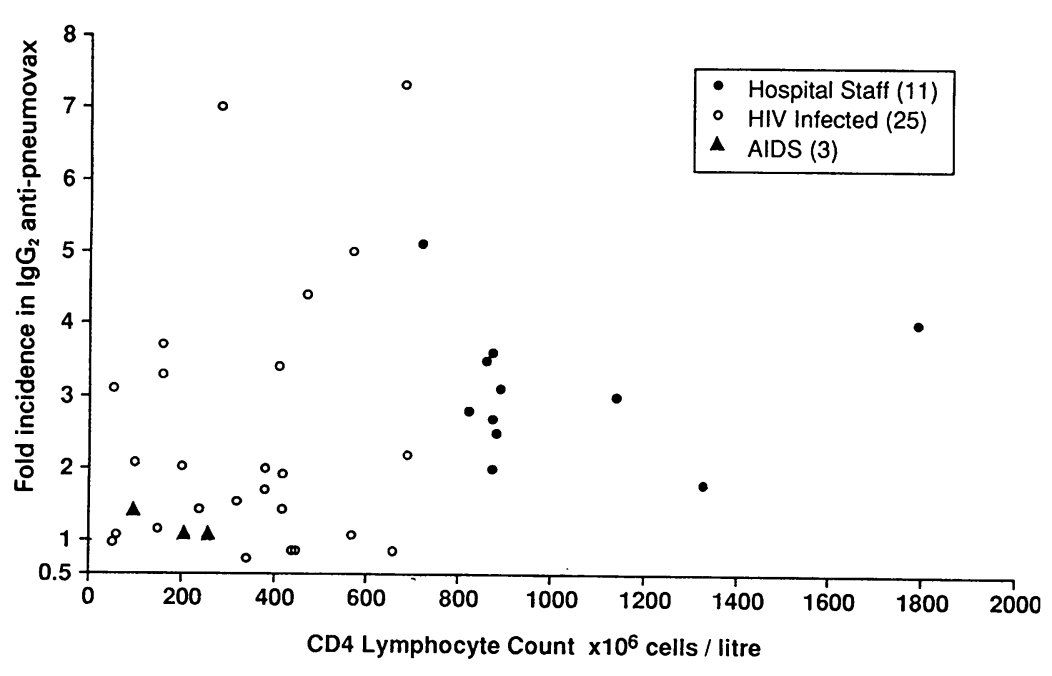

Figure 2 Lack of correlation between pre-immunisation CD4 lymphocyte count and fold increase in IgG2 specific anti-Pneumovax II antibody titre post immunisation. 1.5 fold increase amounts to an increase in specific antibody of 50\%. $(r=0.0285 ; p>0.05)$ were for 2 hours. All dilution and wash steps employed phosphate buffered saline containing $0.05 \%$ tween-20. Patients sera were diluted 1/100. Mouse monoclonal antibodies specific for either IgG1 or IgG2 (The Binding Site, Birmingham UK.), and peroxidase labelled rabbit anti-mouse IgG (Dako Ltd UK.) were each diluted to $1 / 1000$. Where samples gave optical density readings outside the linear range of a standard curve run on each assay, samples were diluted and retested. Results are expressed in ELISA units based on serum from an unimmunised blood donor ascribed values of 50 and 15 units in the IgG2 and IgG1 assays respectively. The coefficients of variation for the $\operatorname{IgG} 2$ and IgG1 assays were $18 \%$ and $16 \%$ respectively. With this in mind and based on our experience immunising normal volunteers, we defined a definite response as one where the post immunisation IgG2 antibody level was $50 \%$ or more higher than the pre immunisation value $(1.5$ fold increase in titre). Pre and post immunisation samples were tested side by side on the same ELISA plate. All sera were tested on three separate occasions and the average values taken.

CD4 counts were measured by the whole blood erythrocyte lysis method ${ }^{13}$ using a flow cytometer (FACScan with simulset software; Becton Dickinson), with mouse monoclonal antibodies (Leu 3/2; Becton Dickinson) to define and enumerate the CD4+, CD8lymphocyte population. Total serum IgG1 and IgG2 levels were measured by single radial diffusion in plates obtained from The Binding Site UK. Results are expressed in grams/litre.

\section{Data analysis}

Several of the parameters studied were not normally distributed. We therefore used non parametric methods of statistical analysis where appropriate. Geometric means are quoted in the figures and throughout the text. Paired data were analysed using the Wilcoxon's signed-rank test. Non paired data were analysed using the Mann-Whitney $U$ Test. Correlation between sets of non parametric data used Spearman's rank analysis.

\section{Results}

A wide range of pre-immunisation IgG2 antiPneumovax antibody levels was seen in all three groups (fig 1). None of the HIV group had proven pneumococcal infections in the 6 months preceding immunisation to account for the high antibody levels seen in a minority. Some of the blood donors similarly had high levels of IgG2 specific antibody. Nor were high IgG2 antibody levels pre immunisation in the HIV group related to stage of disease or CD4 count.

Significantly higher levels of IgG2 antibody were found post immunisation for both the hospital staff $(T=0 ; p<0.001)$, and the HIV group $(T=34 ; p<0.01)$. However, the magnitude of the response was significantly 


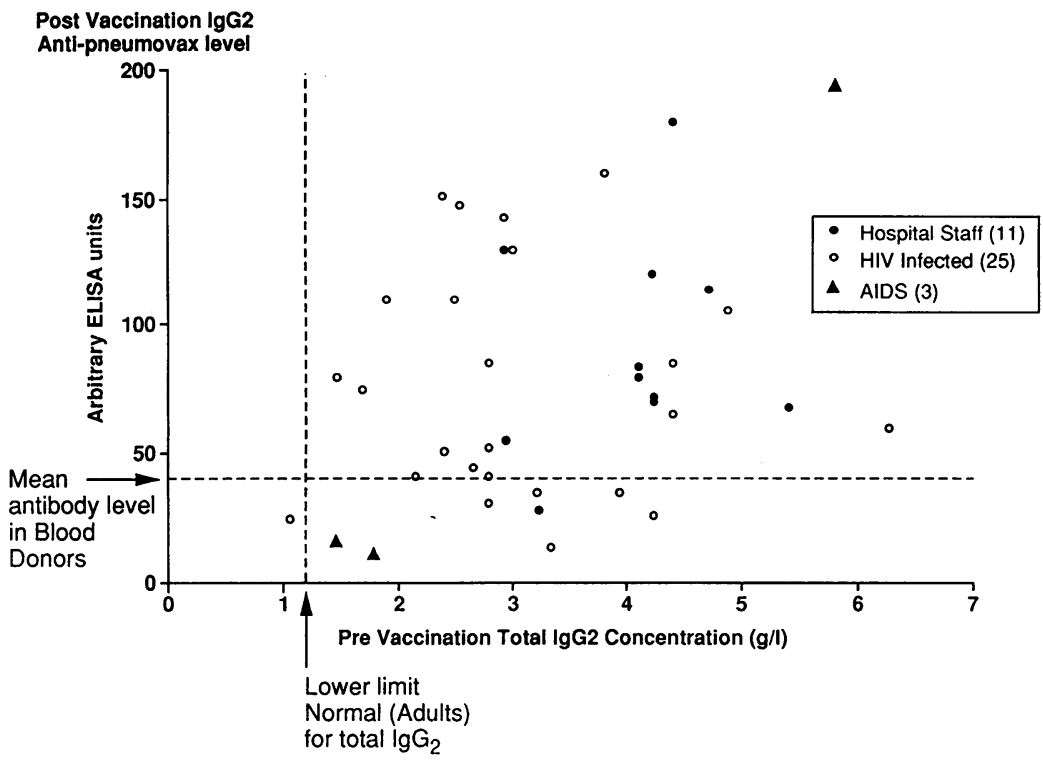

Figure 3 Lack of correlation between the post-immunisation titre of specific IgG2 anti-Pneumovax II and the pre-immunisation total serum IgG2 level ( $r=0.26622$; $p>0.05$ ).

\section{Discussion}

Our results, in agreement with those of Ballet et $a l^{11}$ indicate that approximately $50 \%$ of persons infected with HIV fail to mount IgG2 responses to PPPV. Definition of what constitutes an adequate response is difficult. Our ELISA assay will detect both high and low affinity antibody, and data on how much IgG2 antibody is required for protection in man are not available. Failure to elevate antibody levels post immunisation can be regarded as a failed response. Taking into account the $18 \%$ coefficient of variation for our assay, an increase of IgG2 specific antibody level of $50 \%$ or more was regarded as a definite response. Only 14/28 HIV patients responded. There was no evidence that any of the HIV infected subgroups disproportionately accounted for the poor responders. Suspicion that responses were better in the haemophiliac group did not reach statistical significance, and in any case, they were immunised subcutaneously rather than intra-muscularly in contrast to the other groups. Poor responses were independent of CD4 counts at time of immunisation. Although PPPV are composed of $\mathrm{T}$ cell independent antigens, the CD4 count was of relevance in this study as a measure of HIV disease progression. Poor responders show no subclass switch to IgG1. The reason for the poor responses in the HIV group is unclear. Absence of IgG2 specific responses raise doubts over whether PPPV will provide protection in up to $50 \%$ of HIV seropositives.

Our assay has some limitations. For example, where an immune response was demonstrated, we did not check whether the antibodies produced covered all the serotypes present in the vaccine. However, this may actually have caused us to overestimate the number of persons mounting adequate responses. IgM, and IgA antibodies are produced alongside IgG2 antibodies, but as already discussed it is the IgG2 isotype that is believed to be crucial in providing protection. Our decision not to study the other isotypes does not therefore detract from our conclusions. Other authors have studied specific IgM, IgA, and/or total IgG responses to PPPV, and report impaired responses in HIV seropositive individuals. ${ }^{10}$

There are three prerequisites for a successful immunisation programme. A vaccine of proven efficacy, a target group significantly at risk of infection, and ability of the target group to mount an effective immune response. Our results and those of Ballet et al. ${ }^{11}$ suggest that in HIV antibody positive patients, the third requirement is not satisfied. A large recent study ${ }^{14}$ confirmed PPPV efficacy in immunocompetent hospital patients aged less than 55 years, but data in age matched immunoincompetent patients were less convincing (HIV patients were excluded from this study). Until similar studies in HIV seropositive are performed, the value of immunising these individuals must remain in doubt. 
1 Simberkoff MS, El Sadr W, Schiffman G, Rahal JJ. Streptococcus pneumoniae infections and bacteremia in patients with Acquired Immunodeficiency Syndrome, with a report of vaccine failure. Am Rev Resp Dis with a report of

2 Chave P, Bille J, Glauser MP, Francioli P. Diagnosis of pulmonary infections in patients with the human immunodeficiency virus. Eur $\mathcal{F}$ Clin Microbiol Inf Dis 1989;8:123-36.

3 Selwyn PA, Feingold AR, Hartel D, et al. Increased risk of bacterial pneumonia in HIV-infected intravenous drug users without acquired immunodeficiency syndrome. AIDS 1988;2:267-72.

4 Pneumococcal Vaccine. In: Immunization against Infectious Disease. London HMSO 1992; Chapter 14:100-4.

5 Centres for Disease Control. Recommendations of the immunization practices advisory committee: pneumococcal polysaccharide vaccine. $M M W R$ 1988;5: mococcal

6 Jeffries R, Kumaratne DS. (Review). Selective IgG subclass deficiency: quantitation and clinical relevence. Clin Exp Immunol 1990;81:357-67.

7 Shield JP, Strobel S, Levinsky RJ, Morgan G. Immunodeficiency presenting as hypergammaglobulinaemia with IgG2 subclass deficiency. Lancet 1992;340:448-50.
8 Lane PJ, McLennon ICM. Impaired IgG2 anti-pneumococcal antibody responses in patients with recurrent infection and normal IgG2 levels but no IgA. Clin Exp Immunol 1986;65:427-31.

9 Siber GR, Schur PH, Aisenberg AC, Weitzman SA, Schiffman G. Correlation between serum IgG2 concentrations and the antibody response to bacterial polysaccharide antigens. $N$ Eng $\mathcal{F}$ Med 1980;303:178-82.

10 Pinching AJ. (Editorial Review). Antibody responses in HIV infection. Clin Exp Immunol 1991;84:181-4.

11 Ballet J, Sulcebe G, Couderc LJ, et al. Impaired antipneumococcal antibody response in patients with AIDS-related persistent generalised lymphadenopathy. Clin Exp Immunol 1987;68:479-87.

12 Fahey JL, Taylor JM, Detels $R$, et al. The prognostic value of cellular and serologic markers in infection with of cellular and serologic markers in infection with human immunodefic

13 Campbell AC. Cellular investigations in the diagnosis of lymphoid malignancy. In: Gooi HC, Chapel $\mathrm{H}$, eds. Clinical Immunology. A Practical Approach Oxford Univ Press. 1990:139-72.

14 Shapiro ED, Berg AT, Austrian R, et al. The protective effect of polyvalent pneumococcal vaccine. $N$ Eng $\mathcal{F} \mathrm{Med}$ 1991;325:1453-60. 